\title{
Note
}

\section{Storage Conditions and Screening of Koji Molds for Starch Hydrolysis in Dried Unripe Apple Pulp}

\author{
Hiromi Izawa $^{1 *}$, Tsutomu SATO ${ }^{2}$ and Shuzo Fujita ${ }^{1}$ \\ ${ }^{I}$ Department of Nutrition, Aomori University of Health and Welfare, 58-1 Mase, Hamadate, Aomori City, Aomori Prefecture \\ 030-8505, Japan \\ ${ }^{2}$ Akita Konno Co., Ltd., 248 Kariwano, Daisen City, Akita Prefecture 019-2112, Japan
}

Received June 2, 2014 ; Accepted August 28, 2014

In this study, we investigated the use of koji molds for starch hydrolysis in unripe apples. During preliminary assessments of storage conditions, starch was completely broken down within 2 weeks at room temperature. However, in dried unripe apple pulp, starch remained after 7 months of storage at room temperature. Furthermore, we examined the abilities of various Aspergillus species to hydrolyze starch in dried unripe apple pulp; $A$. oryzae showed greater hydrolysis ability than $A$. awamori or $A$. awamori var. kawachii. Subsequently, various $A$. oryzae were inoculated into agar plate medium containing dried unripe apple pulp powder as the only nutritional source. Among the tested koji molds, A. oryzae AOK 139 showed the greatest growth, presumably because of its comparatively high cellulase expression. Because the starch in apples is located within walled cells, we conclude that hydrolysis requires the use of koji molds with high cellulase activity and productivity.

Keywords: koji mold, Aspergillus oryzae, unripe apple, starch, hydrolysis

\section{Introduction}

During the annual Japanese apple cultivation season, good quality apples are abundant and unripe apples are discarded. These unripe apples contain large quantities of starch and represent an unused organic resource (Singh et al., 2005). Although the starch in unripe apples is contained within cells that are protected by the cell walls and is difficult to utilize, starch from barley can be saccharified using koji mold, despite complete or partial coverage with the husk (Shoji et al., 2007, Masuda et al., 2009). Thus, starch hydrolysis in unripe apples may provide a new food material and effectively reclaim a resource. Several types of koji molds can be purchased at specialized shops in Japan. The koji mold Aspergillus oryzae is a well-described fungus that can hydrolyze starch, and is used in the production of several fermented foods including alcoholic beverages and fermented flavoring agents such as soy sauce and miso. In addition, the closely related strains Aspergillus awamori and A. awamori var. kawachii are also used to produce alcoholic beverages. The use of unripe apples as processed food materials will be beneficial for the utilization of unused carbohydrate biomass. Furthermore, the koji molds used can then serve as raw materials for alcoholic beverages, which represent high value-added products. As a result, the effective use of unripe apples would be financially advantageous for apple farmers.

In this study, we assessed starch hydrolysis after storage of unripe apples, and then screened koji molds for hydrolysis efficacy in nonperishable dried apple pulp.

\section{Materials and Methods}

Samples The unripe apples used in this study were Malus domestica Borkh cultivars, namely Fuji, Kougyoku, Ourin and Sekaiichi. Apples were harvested from a private orchard in Hirosaki, Aomori, Japan, at the end of May 2010. Fruits of each 
cultivar were stored in a warehouse at the orchard at room temperature for approximately $2-4$ days, and were subsequently transferred to a laboratory for experimentation. All dry koji molds were purchased from Akita Konno Co. (Akita, Japan). Rice was purchased from a local grocery store and was used as a positive control.

Storage test Unripe Kougyoku, Ourin, and Sekaiichi apples were used for the storage test. Starch content in the unripe apples was measured using a total starch assay kit (Megazyme International Ireland, Wicklow, Ireland). Unripe apples were separated into two groups and were stored at room temperature or at $4^{\circ} \mathrm{C}$. The presence of starch was measured semi-quantitatively using iodine staining. Staining in cross sections of unripe apples was evaluated as,,++++++ , or "Not Detected". Fuji apples were cut into small pieces, and placed in a juicer to produce unripe apple pulp (GP-E1503; Tribest Corp., Anaheim, CA, USA). Pulps were then dried at $60^{\circ} \mathrm{C}$ for approximately $3-4 \mathrm{~h}$. Dried pulps were stored at room temperature for 7 months. Iodine staining was performed again to determine the presence of starch at the end of storage.

Measurement of $\alpha$-amylase activity Evaluation of $\alpha$-amylase activity followed the procedure described by Yoshizaki et al. (Yoshizaki et al., 2010) with minor modifications. In brief, dried unripe Fuji apple pulp ( $2 \mathrm{~g}$ ) was immersed in distilled water overnight. Excess moisture was removed and the wet pulp was autoclaved. After cooling, dry koji molds $(0.1 \%$ of wet pulp weight) were inoculated into wet pulp and incubated at $30^{\circ} \mathrm{C}$ for 1 , 3 , or 5 days. Subsequently, $0.1 \% \mathrm{NaCl}$ solution $(50 \mathrm{~mL})$ was added and the pulp was stored overnight at $4^{\circ} \mathrm{C}$. The pulp was then passed through a filter paper and the filtrate was used as a crude extract for $\alpha$-amylase activity assay using an $\alpha$-amylase assay kit (Kikkoman Corp., Tokyo, Japan). Rice was treated in the same way as the pulp and was used as a positive control.

Screening of koji molds Koji molds were screened using the procedure of Yoshizaki et al. (Yoshizaki et al., 2010). Solid-state plates were prepared with unripe apple pulp as follows. Dried unripe apple pulps were ground in a mixer mill (IFM-800DG; Iwatani Corp., Tokyo, Japan) and the resulting powder was dissolved to $6.25 \%$ in distilled water. All media contained 2\% agar. The agar medium was then autoclaved and dispensed into glass dishes to form the solid-state plates. Rice powder (6.67\%) was used instead of unripe apple pulps as the positive control, and starch-free plates were used as the negative control. Solid-state plates were inoculated at the center with $5 \mu \mathrm{L}$ of $5 \%$ dry koji mold suspension. Growth of koji molds on the solid-state plates was assessed by measuring the diameter of mycelial colonies.

Statistical analysis Data are expressed as means \pm SEM. The significance of differences was determined using a one-way analysis of variance followed by Tukey's test.

\section{Results}

The starch contents of unripe Kougyoku, Ourin, and Sekaiichi apples were $3.61-7.70 \mathrm{~g} / \mathrm{g}$ of pulp (Table 1). The persistence of starch in unripe apples was compared at various temperatures, and is presented in Table 1 and Fig. 1A and 1B. At room temperature, starch remained intact for less than $8-12$ days, whereas at $4{ }^{\circ} \mathrm{C}$, starch remained intact for more than 14 days. Moreover, starch remained in unripe apple pulp after 7 months of storage at room temperature, as indicated by iodine staining (Fig. 1C).

The starch hydrolysis by Aspergillus species was assessed on solid-state plates containing dried unripe apple pulps (Fig. 2). Whereas the $\alpha$-amylase activity of $A$. oryzae was significantly higher than that of A. awamori and A. awamori var. kawachii, the $\alpha$-amylase activity of all koji molds was much lower with unripe apple than that with rice.

Ten $A$. oryzae strains were inoculated onto agar plates containing dried unripe apple pulp powder as the only nutritional source (Fig. 3), and A. oryzae for moromi was incubated in rice medium as a positive control. In the presence of apple pulp powder, A. oryzae AOK 139 showed significantly greater growth than the other 10 A. oryzae strains.

\section{Discussion}

Depending on the availability of a consistent supply of unripe apples, novel processed food materials could be produced by the hydrolysis of unripe apple starch. However, unripe apples are only

Table 1. Preservation of starch in unripe apples.

\begin{tabular}{|c|c|c|c|c|c|c|c|}
\hline \multirow{2}{*}{ Cultivar } & \multirow{2}{*}{$\begin{array}{c}\text { Starch content } \\
\text { (g/g of prestorage pulp) }\end{array}$} & \multirow{2}{*}{ Storage temperature } & \multicolumn{5}{|c|}{ Storage period (days) } \\
\hline & & & 1 & 3 & 8 & 12 & 14 \\
\hline \multirow{2}{*}{ Kougyoku } & \multirow{2}{*}{$5.81 \pm 0.01$} & room temperature & ++ & ++ & + & N.D. & N.D. \\
\hline & & $4^{\circ} \mathrm{C}$ & ++ & ++ & ++ & + & + \\
\hline \multirow{2}{*}{ Ourin } & \multirow{2}{*}{$7.70 \pm 0.06$} & room temperature & +++ & ++ & + & N.D. & N.D. \\
\hline & & $4^{\circ} \mathrm{C}$ & +++ & +++ & ++ & ++ & ++ \\
\hline \multirow{2}{*}{ Sekaiichi } & \multirow{2}{*}{$3.61 \pm 0.00$} & room temperature & +++ & + & N.D. & N.D. & N.D. \\
\hline & & $4^{\circ} \mathrm{C}$ & +++ & ++ & ++ & ++ & ++ \\
\hline
\end{tabular}

Starch content values are expressed as means \pm SEM $(n=3) .+++/++/+=$ Iodine staining of unripe apple cross-sections. N.D.; Not Detected. 

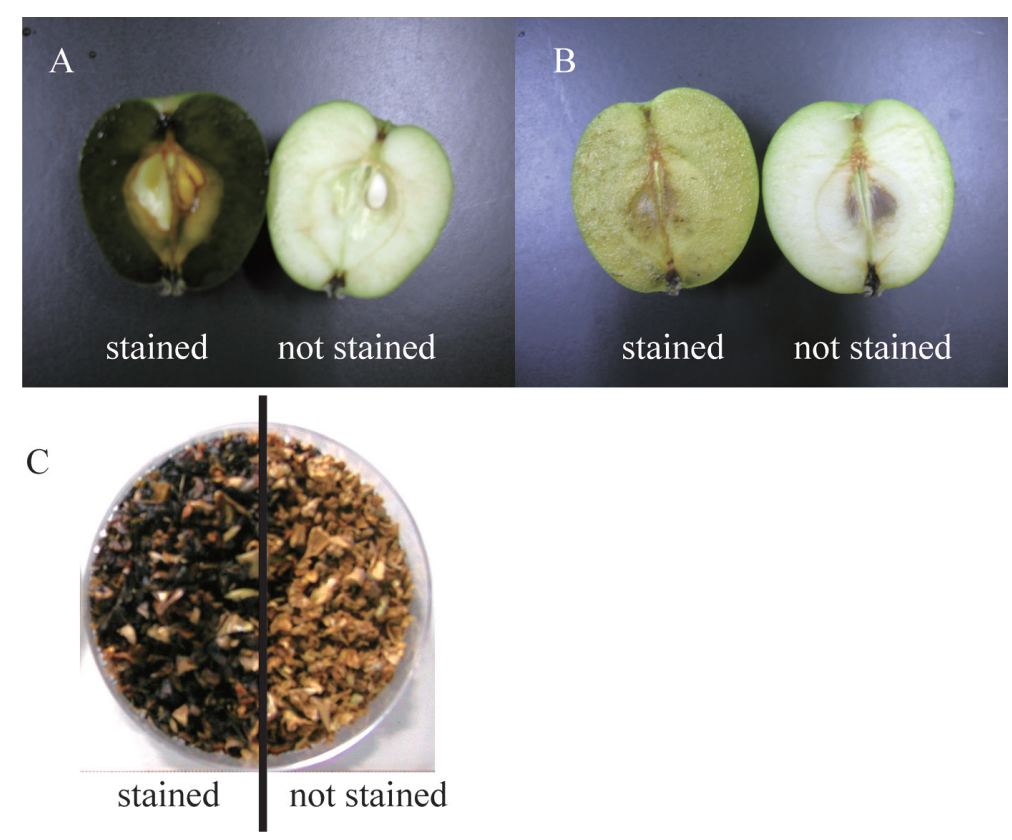

Fig. 1. Iodine staining of unripe apple cross-sections.

Unripe Orin apples were stored for 0 (A) and 14 days (B). Dried unripe Fuji apple pulps were stored for 7 months $(\mathrm{C})$. Both were stored at $25^{\circ} \mathrm{C}$.

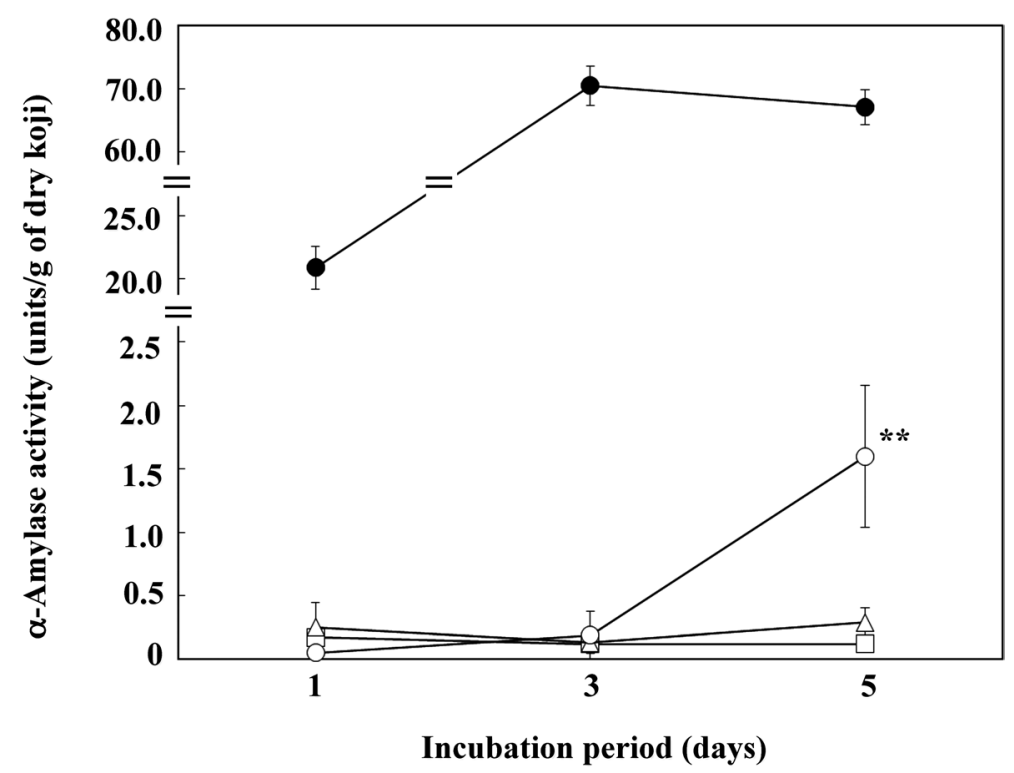

Fig. 2. Comparison of Aspergillus species for starch hydrolysis of dried unripe apple residues.

Open circles: A. oryzae (moromi), open triangles: A. awamori var. kawachii (Sochu white koji fungus), open squares: A. awamori (Sochu black koji), closed circles: $A$. oryzae (moromi, rice-containing medium as a positive control). Data are expressed as means $\pm \operatorname{SEM}(\mathrm{n}=3)$. ** $\mathrm{P}<0.01$ compared with $A$. awamori var. kawachii Sochu white koji fungus or A. awamori Sochu black koji

available between June and July, necessitating storage conditions that preserve starch for its use all year long. In the present study, we investigated storage conditions for starch maintenance in unripe apples, and showed that starch is better preserved in unripe apples stored at $4{ }^{\circ} \mathrm{C}$ than at room temperature. This result is in agreement with that of Carrin et al. (Carrin et al., 2004), who demonstrated that starch content in unripe apples rapidly decreased as the apples ripened and undetectable concentrations were observed after storage of unripe apples for 3 weeks at room temperature. In contrast, we observed that starch in dried unripe apple pulp remained intact for more than 7 months, indicating that dried unripe apples can be stored at room temperature and used all year around.

In subsequent experiments, we identified Aspergillus species capable of starch hydrolysis in dried unripe apples. A. oryzae hydrolyzed dried unripe apples more efficiently than A. awamori or 


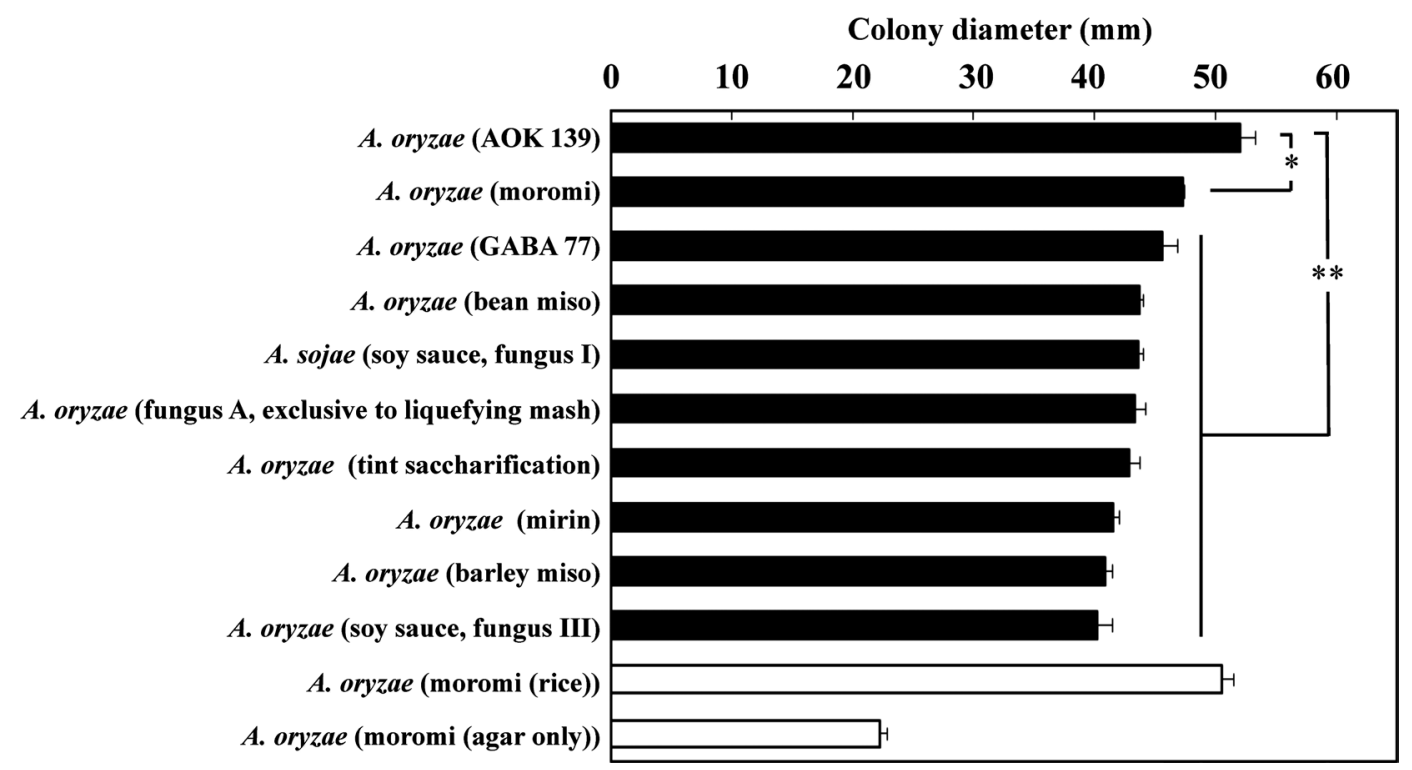

Fig. 3. Screening of Aspergillus species for growth in media containing unripe apple pulp.

Colony diameter was measured at the end of the 5-day incubation period. The apple medium contained $6.25 \%$ dried unripe apple pulp, while the rice medium contained $6.67 \%$ rice powder. All media contained 2\% agar. Data are expressed as means $\pm \operatorname{SEM}(\mathrm{n}=3) . * * \mathrm{P}<0.01 . * \mathrm{P}<0.05$.

A. awamori var. kawachii. However, $\alpha$-amylase activities were much lower in unripe apples than in rice, suggesting that these three koji mold strains may not grow in unripe apples. Koji molds are widely used in the production of sake and/or shochu mash, and usually produce very little cell-wall degrading enzyme. However, other koji molds, such as those used in the production of miso paste or soy sauce, produce large amounts of cell-wall degrading enzymes and may grow in unripe apple cultures. Thus, the growth of nine koji molds was investigated in plate cultures. In these experiments, A. oryzae AOK139 produced the largest colonies on solid-state media. This koji mold, which is mainly used in miso production, exhibits antimutagenic activity and produces free fatty acids, fatty acid ethyl esters, lipase, and cellulase (Watanabe et al., 2004). Because the cellulase from A. oryzae AOK139 degrades the cell walls of unripe apples, this koji mold likely has access to the intercellular starch, as indicated by the prolific growth on solidstate plates containing unripe apple pulp.

In this study, storage conditions that permitted starch retention in unripe apples were investigated, and commercial koji molds were screened for starch hydrolysis efficiency in unripe apples. Starch in dried unripe apple pulp was maintained for more than 7 months at room temperature, suggesting that dried unripe apples could be stored for year-round distribution. Screening of koji molds for starch hydrolysis in dried unripe apple pulp revealed that $A$. oryzae AOK 139 grew the best and may hydrolyze starch effectively in unripe apples.
Acknowledgments This research was funded in part by the Adaptable and Seamless Technology Transfer Program through Target-driven R\&D, the Japan Science and Technology Agency.

\section{References}

Carrín, M. E., Ceci, L., and Jorge, L. (2004) Characterization of starch in apple juice and its degradation by amylases. Food Chem., 87, 173-178.

Masuda, S., Kikuchi, K., Matsumoto, Y., Sugimoto, T., Shoji, H., and Tanabe, M. (2009) Analysis of enzyme production by submerged culture of Aspergillus oryzae using whole barley. Biosci. Biotechnol. Biochem., 73, 2190-2195.

Shoji, H., Sugimoto, T., Hosoi, K., Shibata, K., Tanabe, M., and Kawatsura, K. (2007) Simultaneous production of glucoamylase and acid-stable alpha-amylase using novel submerged culture of Aspergillus kawachii NBRC4308. J. Biosci. Bioeng., 103, 203-205.

Singh, N., Inouchi, N., and Nishinari, K. (2005) Morphological, structural, thermal, and rheological characteristics of starches separated from apples of different cultivars. J. Agric. Food Chem., 53, 10193-10199.

Yoshizaki, Y., Susuki, T., Takamine, K., Tamaki, H., Ito, K., and Sameshima, Y. (2010) Characterization of glucoamylase and alphaamylase from Monascus anka: enhanced production of alpha-amylase in red koji. J. Biosci. Bioeng., 110, 670-674.

Watanabe, T., Owari, K., Hori, K., and Takahashi, K. (2004) Selection of koji mold strain for making functional miso as rich antimutagenic activity. Nippon Shokuhin Kagaku Kogaku Kaishi, 51, 698-702 (in Japanese). 